\title{
Performance Study of Soft Local Binary Pattern over Local Binary Pattern under Noisy Images
}

\author{
Sabina Yasmin, Md. Masud Rana \\ Department of Electrical and Electronic Engineering, \\ Rajshahi University of Engineering \& Technology, Rajshahi-6204, Bangladesh
}

\begin{tabular}{l} 
Article Info \\
\hline Article history: \\
Received Aug 1, 2015 \\
Revised Nov 9, 2015 \\
Accepted Nov 25, 2015 \\
\hline Keyword: \\
Local Binary Pattern \\
Feature Extraction \\
Soft Local Binary Pattern \\
Quality Measurement \\
Filtering Methods
\end{tabular}

Corresponding Author:

Sabina Yasmin, Department of Electrical and Electronic Engineering, Rajshahi University of Engineering \& Technology, Rajshahi 6204, Bangladesh. Email: syasmin.ruet@gmail.com

\begin{abstract}
In this paper, the performance of soft local binary pattern (SLBP) method has been investigated with edge detection techniques for face recognition in case of noisy condition. Various edge detection techniques such as Canny, Robert and Log methods have been used with SLBP for recognizing faces. The results obtained using SLBP with various edge detection for noisy condition based on image quality measurement shows better recognition rate compared to the results obtained using local binary pattern (LBP). Simplified edge detection methods simplify the images as a result SLBP with edge detection requires less computation time compared with edge detection of LBP.
\end{abstract}

Copyright (c) 2016 Institute of Advanced Engineering and Science. All rights reserved.

\section{INTRODUCTION}

Face recognition face detection and facial expression recognition have been an interesting area of research over the past few years as a result of its growing usage in many applications in fields such as airlines, banking, security, multimedia applications etc. Each individual can be identified and verified by this method. Actually Face Recognition system works by comparing unknown facial image with known individuals from a large database and then the system returns a value of similarity measurement between the two images [1]-[9]. The Facial image preprocessing is very important part of face recognition or face detection system. One of the vital preprocessing tasks is image denoising. Image preprocessing is the technique of enhancing data images prior to computational processing [2]. There are different types of image noises which degrade the image quality and also have the filtering techniques for removing those noises. An efficient denoising technique should completely remove noises without affecting the image quality. Also we have many quality matrices to measure image quality [2]-[4]. MSE, PSNR, and SSIM are some useful and most commonly used image quality metrics in image processing [3]. The SSIM index can be viewed as a quality measure of one of the images being compared provided the other image is regarded as of perfect quality [10]. Using the different quality metrics we can easily measure the quality after denoising the noisy image and after that we can compare the differences among them.

Various methods have been used for extracting the useful features from face images to face recognition. Among the methods LBP is most efficient for Face recognition. The local binary pattern (LBP) that is invariant to monotonic gray-scale transformations which is very important for texture analysis. With LBP it is possible to describe the texture and shape of a digital image [1]-[2]. This is done by dividing an image into several small regions from which the features are extracted (Figure 1). Face recognition system 
using Local Binary Pattern (LBP) was introduced in 1996 by Ojala et al. [11]. The LBP operator is one of the best performing texture descriptors and it has been widely used in various applications. This operator works with the eight neighbors of the center pixel considering as a threshold [8]-[11]. The LBP is invariant to monotonic gray-scale transformations which is very important for texture analysis. With LBP it is possible to describe the texture and shape of a digital image [8]-[9]. LBP has been improved with multiscale and different texture patterns such as uniform, rotation invariant and rotation invariant uniform patterns [11]-[14].

The major disadvantages of LBP is that it is not robust that is sensitive to noise and another is a small change in the input image would always cause only a small change in the output. But in soft histogram version, one pixel typically contributes to more than one bin [15]-[16]. Soft Local Binary Pattern is more efficient than LBP in case of noisy images [15]-[16].

In our study we have compared different noisy images and after removing the noise using different filtering methods for both LBP and SLBP for face recognition. Soft Local Binary Pattern can recognize the faces while the image is noisy but LBP could not that means recognition rate is very poor. In section 2, edge detection techniques, image quality measurement techniques have been described that has been used with SLPB and LPB method to measure the quality in section 3. Next, in Section 4 and 5, LBP and SLBP methods have been described. Finally, numerical validation of SLBP method compared with LBP is provided in Section 6.

\section{EDGE DETECTION TECHNIQUES}

Edge detection is a basic tool used in image processing, basically for feature detection and extraction, which aim to identify points in a digital image where brightness of image changes sharply and find discontinuities. The purpose of edge detection is significantly reducing the amount of data in an image and preserves the structural properties for further image processing. Different edge detection techniques are mentioned below:

\subsection{Sobel Operator}

The Sobel operator performs a 2-D spatial gradient measurement on an image and so emphasizes regions of high spatial frequency that correspond to edges. One kernel is simply the other rotated by $90^{\circ}$. Calculate the edges in both horizontal and vertical directions. Then combine the information into a single metric.

\subsection{Robert Operator}

The Roberts operator performs a simple, quick to compute, 2-D spatial gradient measurement on an image. Pixel values at each point in the output represent the estimated absolute magnitude of the spatial gradient of the input image at that point [17].

\subsection{Prewitt Operator}

The prewitt edge detector is an appropriate way to estimate the magnitude and orientation of an edge. The prewitt operator is limited to 8 possible orientations. This gradient based edge detector is estimated in the 3x3 neighbourhood for eight directions. All the eight convolution masks are calculated. One convolution mask is then selected, namely that with the largest module [17].

\subsection{Canny Operator}

Canny operator is based on three criteria. The Canny Edge Detection Smoothes the image with a Gaussian filter. Then compute the gradient magnitude and orientation using finite-difference approximations for the partial derivatives. And finally apply non-maxima suppression to the gradient magnitude, use the double thresholding algorithm to detect edges [18].

\subsection{LoG Operator}

The Laplacian method searches for zero crossings in the second derivative of the image to find edges. Laplacian of Gaussian method combines Gaussian filtering with the Laplacian for edge detection. After calculating second-order derivative of an image, the value of a point which is greater than a specified threshold and one of its neighbors is less than the negative of the threshold is called zero-crossing [18].

\section{IMAGE QUALITY MEASUREMENT}

In recent years, a lot of developments have been made to measure image quality that correlate with perceived quality. Image quality is a characteristic of an image that measures the perceived image 
degradation [4]. This degradation of image by noise is some random error. This Noise could be added along with the image during capturing, transmitting or during the processing [6]. We have applied different noises in images and removed the noises using different filtering techniques and compare using MSE, PSNR, and SSIM which are commonly used image quality metrics in image processing.

\subsection{Mean Square Error (MSE)}

This measurement is used to compute an error signal by subtracting the test signal from the original, and then computing the average energy of the error. The mean square error (MSE) is the simplest, and the most widely used image quality measurement. MSE for two $\mathrm{P} \times \mathrm{Q}$ monochrome images, $\mathrm{A}$ is the original image and $\mathrm{B}$ is the distorted image and also $\mathrm{m}, \mathrm{n}$ are the width and height of the image. Then the MSE is defined as the following [3],[7].

$$
M S E=\frac{1}{P Q} \sum_{m=1}^{P} \sum_{n=1}^{Q}[A(m, n)-B(m, n)]^{2}
$$

\subsection{Root Mean Square Error (RMSE)}

The Root Mean Square Error (RMSE) is the square root of MSE [3],[7].

$$
R M S E=\sqrt{\frac{1}{P Q} \sum_{m=1}^{P} \sum_{n=1}^{Q}[A(m, n)-B(m, n)]^{2}}
$$

\subsection{Peak Signal to Noise Ratio (PSNR)}

The Peak Signal to Noise Ratio (PSNR) represents the value of the noisy image with respect to the original image. The PSNR is evaluated in decibels and is inversely proportional with the Mean Square Error is defined as the following [3],[7].

$$
P S N R=10 \log 10\left(\frac{L^{2}}{M S E}\right)
$$

Where $\mathrm{L}$ is the dynamic range of the pixel values.

\subsection{Structural Similarity Index Measurement (SSIM)}

The Structural Similarity Index Measurement (SSIM) is another useful method for measuring the similarity between two images. The SSIM index can be viewed as a quality measure of one of the images being compared provided the other image is regarded as of perfect quality. The SSIM is defined as the following [3],[10].

$$
\operatorname{SSIM}(x, y)=[l(x, y)]^{\alpha}[c(x, y)]^{\beta}[s(x, y)]^{\gamma}
$$

Where $\alpha>0, \beta>0$ and $\gamma>0$ control the relative significance of the three terms of the index. The luminance, contrast and structural components of the index.

\section{LOCAL BINARY PATTERN}

Face recognition system using Local Binary Pattern (LBP) was introduced in 1996 by Ojala et al. [8]-[12]. The LBP operator is one of the best performing texture descriptors and it has been widely used in various applications. In this paper we have implemented different edge detection techniques such as sobel, prewitt, robert, canny and log as preprocessing before applying LBP in order to get better performance of LBP. The process of face recognition using LBP edge detection is given in Figure 1. 


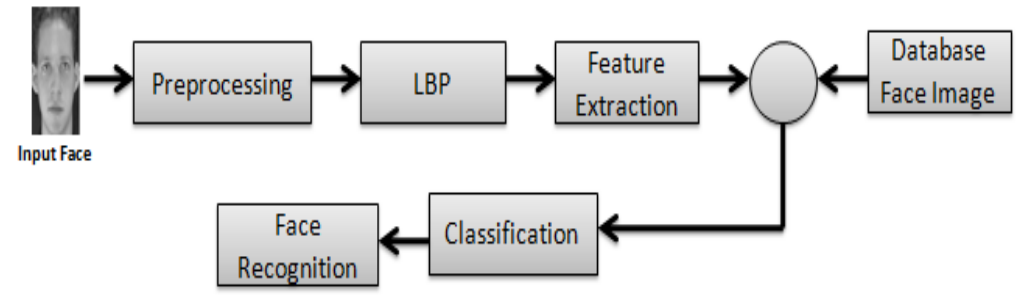

Figure 1. Process of Face Recognition using LBP [1]

Noise elimination and edge detection are used as preprocessing in LBP. This LBP operator works with the eight neighbors of the center pixel considering as a threshold. If a neighbor pixel has a higher gray value than the center pixel (or the same gray value) than a one is assigned to that pixel, otherwise zero. The center value is constructed by concatenating the binary numbers from top left corner in clockwise direction. And finally the decimal value is produced by multiplying the threshold values with weights given to the corresponding pixels and summing up the result, is called LBP codes [15] as shown in Figure 2.

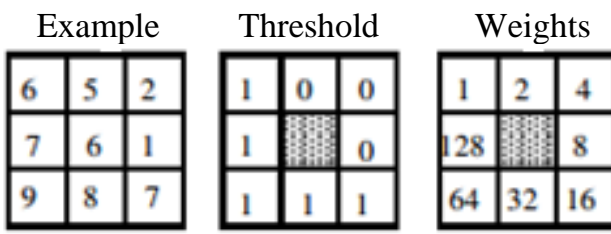

Binary Pattern $=1000111$

Decimal $=1+16+32+64+128=241$

Figure 2. Calculating the original LBP code

Formally, then the resulting LBP can be expressed in decimal form defined by the following

$$
L B P_{P, R}\left(x_{c}, y_{c}\right)=\sum_{P=0}^{P-1} s\left(i_{p}-i_{c}\right) 2^{P}
$$

where $i_{C}$ and $i_{P}$ are, respectively, gray-level values of the central pixel and $P$ surrounding pixels in the circle neighborhood with a radius $\mathrm{R}$ of given pixel at $\left(\mathrm{x}_{\mathrm{c}}, \mathrm{y}_{\mathrm{c}}\right)$, and function $\mathrm{s}(\mathrm{x})$ is defined as [14].

$$
s(x)= \begin{cases}1, & \text { if } x \geq 0 \\ 0, & \text { if } x<0\end{cases}
$$

\section{SOFT LOCAL BINARY PATTERN (SLBP)}

A drawback of the local binary pattern is not robust that is a small change in the input image would always cause only a small change in the output. It is sensitive to noise. But in soft histogram version, one pixel typically contributes to more than one bin [15],[16]. To increase the robustness of the operator SLBP propose the thresholding function $s(x)$ is replaced by the following two fuzzy membership functions:

$$
f_{1, d}(z)=\left\{\begin{array}{cc}
0, & z<-d \\
0.5+0.5 \frac{z}{d}, & -d \leq z \leq d \\
1 & z>d
\end{array}\right.
$$




$$
f_{0, d}(z)=1-f_{1, d}(z)
$$

The parameter d controls the amount of fuzzification the function performs. These two fuzzification functions are plotted below:

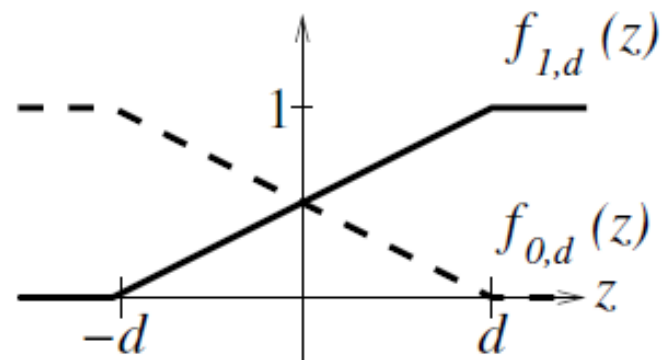

Figure 3. The two fuzzification functions $f_{0, d}$ and $f_{1, d}$

\section{NUMERICAL VALIDATION OF SLBP AND LBP}

In analysis of LBP and SLBP we have compared that if we use edge detection method prior to apply recognition system then the efficiency will be enhanced. So we have used some edge detection methods and compared the results. From Table 1 to Table 5 we have shown the comparative data between LBP and SLBP. In paper [16] mean classification error rates were compared with LBP and SLBP, paper [13] showed the face recognition rate of LBP with different parameters. But in our experiment we have discussed about the face recognition comparison rate between LBP and SLBP.

For analysis we have used ORL database [5] where ten different images of 256 gray levels with a resolution of 92x112 pixels of each of 40 distinct persons. In this experiment we process the sample image with different edge detection methods then calculate the LBP after that compare using Chi Square method from database face images to measure face recognition rate and the same manner is used for SLBP. Different edge detection methods are used first then the different noises are applied and check the recognition rate. After removal of noises the recognition rate is analized. Sample image of original and noisy are depict below:

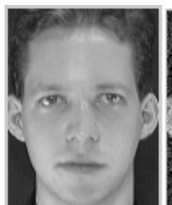

(a)

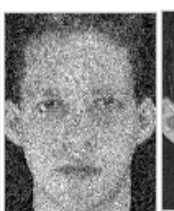

(b)

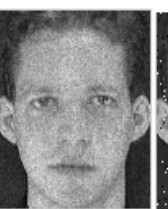

(c)

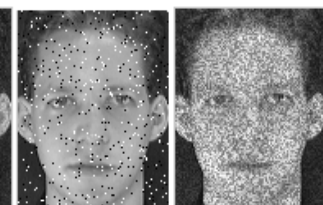

(d) (e)

Figure 4. The original image(a) and noisy image (Gaussian-(a), Poisson(b), Salt \& Pepper (c) and Speckle(d))

Table 1. Comparison of Sobel edge detection for LBP and SLBP

\begin{tabular}{clccccc}
\hline LBP(1,8) & Noise Methods & $\begin{array}{c}\text { Recognition Rate } \\
\text { of noisy image }\end{array}$ & $\begin{array}{c}\text { Rate after } \\
\text { Denoising }\end{array}$ & SLBP & $\begin{array}{c}\text { Recognition Rate } \\
\text { of noisy image }\end{array}$ & $\begin{array}{c}\text { Rate after } \\
\text { Denoising }\end{array}$ \\
\hline \multirow{3}{*}{$93.98 \%$} & Gaussian & $54.57 \%$ & $75.59 \%$ & & $81.61 \%$ & $83.62 \%$ \\
& Salt \& Pepper & $32.25 \%$ & $82.92 \%$ & $89.32 \%$ & $81.61 \%$ & $84.98 \%$ \\
& Poisson & $81.95 \%$ & $83.78 \%$ & $88.57 \%$ & $89.84 \%$ \\
& Speckle & $34.34 \%$ & $73.91 \%$ & & $67.44 \%$ & $75.01 \%$ \\
\hline
\end{tabular}

Using Sobel edge detection face recognition rate is $93.98 \%$ for LBP and $89.32 \%$ for SLBP. But if the images are noisy with different techniques then recognition rate will be reduced and after removing the noises the recognition rate are enhanced. Table 1 shows the comparison data. 
Table 2. Comparison of Canny edge detection for LBP and SLBP

\begin{tabular}{clccccc}
\hline \multirow{2}{*}{ LBP(1,8) } & $\begin{array}{c}\text { Noise } \\
\text { Methods }\end{array}$ & $\begin{array}{c}\text { Recognition Rate of } \\
\text { noisy image }\end{array}$ & $\begin{array}{c}\text { Rate after } \\
\text { Denoising }\end{array}$ & SLBP & $\begin{array}{c}\text { Recognition Rate } \\
\text { of noisy image }\end{array}$ & $\begin{array}{c}\text { Rate after } \\
\text { Denoising }\end{array}$ \\
\hline \multirow{3}{*}{$91.42 \%$} & Gaussian & $0 \%$ & $55.76 \%$ & & $4.09 \%$ & $63.28 \%$ \\
& Salt \& Pepper & $68.42 \%$ & $76.26 \%$ & \multirow{2}{*}{$91.58 \%$} & $79.57 \%$ & $84.42 \%$ \\
& Poisson & $70.38 \%$ & $82.58 \%$ & & $79.07 \%$ & $88.24 \%$ \\
& Speckle & $0 \%$ & $17.08 \%$ & & $4.3 \%$ & $24 \%$ \\
\hline
\end{tabular}

In Table 2 Canny edge detection method is used and has got the comparison table of fresh image recognition rate of LBP and SLBP, rate of noisy image and noise removed image respectively. The data shows that SLBP recognition rate is higher than LBP even for the noisy images.

Table 3. Comparison of Robert edge detection for LBP and SLBP

\begin{tabular}{clccccc}
\hline \multirow{2}{*}{ LBP(1,8) } & $\begin{array}{c}\text { Noise } \\
\text { Methods }\end{array}$ & $\begin{array}{c}\text { Recognition Rate of } \\
\text { noisy image }\end{array}$ & $\begin{array}{c}\text { Rate after } \\
\text { Denoising }\end{array}$ & SLBP & $\begin{array}{c}\text { Recognition Rate } \\
\text { of noisy image }\end{array}$ & $\begin{array}{c}\text { Rate after } \\
\text { Denoising }\end{array}$ \\
\hline \multirow{4}{*}{$88.93 \%$} & Gaussian & $0 \%$ & $43.78 \%$ & & $22.79 \%$ & $66.18 \%$ \\
& Salt \& Pepper & $0.35 \%$ & $73.35 \%$ & \multirow{2}{*}{$92.48 \%$} & $55.67 \%$ & $84.19 \%$ \\
& Poisson & $34.66 \%$ & $67.10 \%$ & & $67.18 \%$ & $88.57 \%$ \\
& Speckle & $0 \%$ & $27.56 \%$ & & $60.86 \%$ & $78.76 \%$ \\
\hline
\end{tabular}

In Table 3 Robert edge detection method is used and the recognition rate of SLBP is higher than LBP, SLBP shows the better recognition rate of in case of noisy image and noise removed images.

Table 4. Comparison of Prewitt edge detection for LBP and SLBP

\begin{tabular}{clccccc}
\hline LBP(1,8) & Noise Methods & $\begin{array}{c}\text { Recognition Rate } \\
\text { of noisy image }\end{array}$ & $\begin{array}{c}\text { Rate after } \\
\text { Denoising }\end{array}$ & SLBP & $\begin{array}{c}\text { Recognition Rate } \\
\text { of noisy image }\end{array}$ & $\begin{array}{c}\text { Rate after } \\
\text { Denoising }\end{array}$ \\
\hline \multirow{3}{*}{$92.15 \%$} & Gaussian & $60.47 \%$ & $76.78 \%$ & & $48.48 \%$ & $84.01 \%$ \\
& Salt \& Pepper & $2.08 \%$ & $82.89 \%$ & $88.27 \%$ & $3.9 \%$ & $92.25 \%$ \\
& Poisson & $73.03 \%$ & $81.39 \%$ & $70.33 \%$ & $87.93 \%$ \\
& Speckle & $43.74 \%$ & $75.33 \%$ & & $76.88 \%$ & $82.40 \%$ \\
\hline
\end{tabular}

In Table 4 Prewitt edge detection method is used and the recognition rate of LBP is higher than SLBP, But SLBP shows the better recognition rate of in case of noisy image and noise removed images.

Table 5. Comparison of Log edge detection for LBP and SLBP

\begin{tabular}{clccccc}
\hline \multirow{2}{*}{ LBP(1,8) } & Noise Methods & $\begin{array}{c}\text { Recognition Rate } \\
\text { of noisy image }\end{array}$ & $\begin{array}{c}\text { Rate after } \\
\text { Denoising }\end{array}$ & SLBP & $\begin{array}{c}\text { Recognition Rate } \\
\text { of noisy image }\end{array}$ & $\begin{array}{c}\text { Rate after } \\
\text { Denoising }\end{array}$ \\
\hline \multirow{3}{*}{$88.87 \%$} & Gaussian & $0 \%$ & $53.76 \%$ & & $0.5 \%$ & $65.17 \%$ \\
& Salt \& Pepper & $57.80 \%$ & $80.03 \%$ & $90.10 \%$ & $68.84 \%$ & $84.49 \%$ \\
& Poisson & $65.59 \%$ & $80.65 \%$ & & $88.33 \%$ & $90.14 \%$ \\
& Speckle & $0 \%$ & $18.85 \%$ & & $66.72 \%$ & $87.74 \%$ \\
\hline
\end{tabular}

In Table 5 Log edge detection method is used and the recognition rate of SLBP is higher than LBP, SLBP shows the better recognition rate of in case of noisy image and noise removed images.

\section{CONCLUSION}

In this paper, we have analyzed soft local binary pattern (SLBP) method with edge detection techniques for face recognition in case of noisy condition. It is seen that if edge detection method is used before applying LBP or SLBP, the time complexity will be reduced. It is also observed that some edge detection techniques showed better result for face recognition using LBP and SLBP. Soft Local Binary Pattern with edge detection methods such as Canny, Robert and Log show the better result with compare to LBP. But in case of noisy images, LBP with edge detection methods provides less efficient result whereas SLBP provides better result for noisy images. 


\section{REFERENCES}

[1] S. Yasmin and M. Rana, "Efficient Local Binary Pattern with Modified Edge Detection Technique for Face Recognition," in Proceeding of International Conference on Electrical Engineering and Information Communication Technology (ICEEICT), May, 21-23, Dhaka, Bangladesh, 2015.

[2] S. Yasmin and M. Rana, "Comparative Study of Denoising Techniques for Facial Image using Quality Measurement," in Proceeding of International Conference on Electrical Engineering and Information Communication Technology (ICEEICT), May, 21-23, Dhaka, Bangladesh, 2015.

[3] Yusra A., et al., "Comparison of Image Quality Assessment: PSNR, HVS, SSIM, UIQI,” International Journal of Scientific \& Engineering Research, vol/issue: 3(8), 2012. ISSN 2229-5518.

[4] J. Patil and S. Jadhav, “A Comparative Study of Image Denoising Techniques,” International Journal of Innovative Research in Science, Engineering and Technology, vol/issue: 2(3), 2013.

[5] http://www.cl.cam.ac.uk/Research/DTG/attarchive/pub/data/att_faces.

[6] Vijayalakshmi. A, et al., "Image Denoising for different noise models by various filters: A Brief Survey," International Journal of Emerging Trends \& Technology in Computer Science (IJETTCS), vol/issue: 3(6), 2014. ISSN 2278- 6856.

[7] C. Srivastava, et al., "Performance Comparison of Various Filters and Wavelet Transform for Image De-Noising," IOSR Journal of Computer Engineering (IOSR-JCE), vol/issue: 10(1), pp. 55-6, 2013. e-ISSN: 2278-0661, pISSN: 2278-8727.

[8] T. Ahonen, et al., "Face recognition with Local Binary Patterns," Machine Vision Group, University of Oulu, Finland, 2004.

[9] T. Ahonen, et al., "Face description with Local Binary Patterns: Application to Face Recognition,” Machine Vision Group, University of Oulu, Finland, 2006.

[10] R. Dosselmann and X. D. Yang, “A Formal Assessment of the Structural Similarity Index,” Technical Report TRCS 2008-2 September, 2008.

[11] M. Ojala, et al., "A comparative study of texture measures with classification based on feature distributions," Pattern Recognition, vol. 29, pp. 51-59, 1996.

[12] T. Ojala, et al., "Multiresolution gray-scale and rotation invariant texture classification with local binary patterns," IEEE Trans. Pattern Anal, Mach. Intell., vol/issue: 24(7), pp. 971-987, 2002.

[13] Anagha V. M., et al., “A New Technique for LBP Method to Improve Face Recognition,” International Journal of Emerging Technology and Advanced Engineering, vol/issue: 1(1), 2011. Website: www.ijetae.com (ISSN 22502459).

[14] T. Ahonen, et al., "Rotation Invariant Image Description with Local Binary Pattern Histogram Fourier Features," A.-B. Salberg, J.Y. Hardeberg, and R. Jenssen (Eds.), Springer-Verlag Berlin Heidelberg, pp. 61-70, 2009.

[15] D. Huang, et al., "Local Binary Patterns and Its Application to Facial Image Analysis: A Survey,”.

[16] T. Ahonen and M. Pietik"ainen, "Soft Histograms for Local Binary Patterns," Machine Vision Group, Infotech Oulu, WWW home page: http://www.ee.oulu.fi/mvg.

[17] N. Senthilkumaran and R. Rajesh, "Edge Detection Techniques for Image Segmentation - A Survey,” Proceedings of the International Conference on Managing Next Generation Software Applications (MNGSA-08), pp.749-760, 2008.

[18] G. T. Shrivakshan, and C. Chandrasekar, "A Comparison of various Edge Detection Techniques used in Image Processing,” IJCSI International Journal of Computer Science Issues, vol/issue: 9(5), 2012. ISSN (Online): 16940814. 